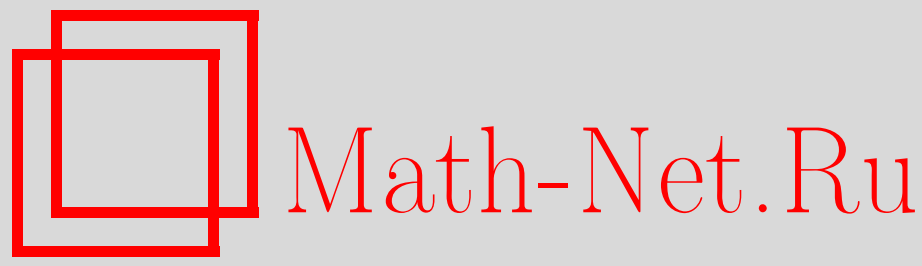

Н. А. Славнов, Интегральные операторы с обобщенным синус-ядром на вещественной оси, ТМФ, 2010, том 165, номер 1, 32-47

DOI: https://doi.org/10.4213/tmf6562

Использование Общероссийского математического портала Math-Net.Ru подразумевает, что вы прочитали и согласны с пользовательским соглашением http://www . mathnet.ru/rus/agreement

Параметры загрузки:

IP : 54.224 .60 .19

26 апреля 2023 г., $17: 14: 55$

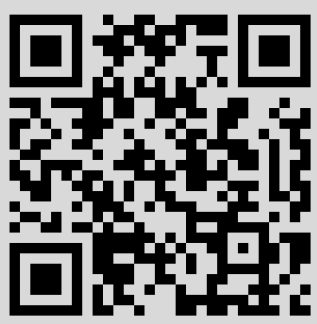




\title{
ИНТЕГРАЛЬНЫЕ ОПЕРАТОРЫ С ОБОБЩЕННЫМ СИНУС-ЯДРОМ НА ВЕЩЕСТВЕННОЙ ОСИ
}

\begin{abstract}
Изучаются асимптотические свойства интегральных операторов с обобщенным синус-ядром, действующих на вещественной оси. В пределе большого $x$ получены формулы для детерминанта Фредгольма и резольвенты. Рассмотрены применения полученных результатов к теории интегрируемых моделей.
\end{abstract}

Ключевые слова: детерминант Фредгольма, резольвента, асимптотическое разложение.

\section{1. ВВЕДЕНИЕ}

Интегральные операторы с синус-ядром возникают во многих областях математической физики. Синус-ядро имеет вид

$$
S(\lambda, \mu)=\frac{\sin \frac{x}{2}(\lambda-\mu)}{\pi(\lambda-\mu)},
$$

где $\lambda$ и $\mu$ суть переменные интегрирования, $x$ - вещественный параметр. Оператор $I+\gamma S$, где $I$ - единичный оператор, а $\gamma$ - некоторое комплексное число, действует на интервале (или системе интервалов) $\ell$ вещественной оси на функции из $L_{2}(\ell)$.

Детерминант Фредгольма интегрального оператора $I-S$ возникает в теории случайных матриц [1]. В скейлинговом пределе $\operatorname{det}_{\ell}(I-S)$ задает вероятность того, что матрица, принадлежащая гауссову унитарному ансамблю, не имеет собственных значений в интервале $x \ell[2]$. Ядро (1.1) также появляется в теории квантовых интегрируемых систем. В частности, детерминант $\operatorname{det}_{\ell}(I+\gamma S)$ описывает различные корреляционные функции при нулевой температуре в модели непроницаемого Бозе-газа [3], [4].

В упомянутых выше интерпретациях синус-ядра наиболее интересный вопрос связан с поведением детерминанта $\operatorname{det}_{\ell}(I+\gamma S)$ при большом $x$. Эта проблема изучалась в многочисленных работах (см. работы [1], [2], [5]-[13]). Очень красивая связь между синус-ядром и уравнением Пенлеве V была исследована в [4], [14]. Методы

*Математический институт им. В. А. Стеклова РАН, Москва, Россия. E-mail: nslavnov@mi.ras.ru 
асимптотического анализа $\operatorname{det}_{\ell}(I+\gamma S)$ основаны на том, что при большом $x$ ядро $S$ становится быстроосциллирующим. Отметим, однако, что это ядро не имеет седловых точек, а сдвиги контура интегрирования не делают его экспоненциально малым. Поэтому стандартные методы асимптотических оценок осциллирующих интегралов в этом случае не работают.

В литературе известны многочисленные обобщения ядра (1.1), сохраняющие его осциллирующую структуру. Одно из таких обобщений возникает в теории обрезанных операторов Винера-Хопфа [15], [16], где интегральный оператор действует на $\mathbb{R}$, а комплексное число $\gamma$ заменяется на некоторую функцию $\gamma(\lambda)$. Другие обобщения синус-ядра в основном использовались для описания корреляционных функций матричных моделей или квантовых интегрируемых систем, эквивалентных свободным фермионам (см. работы [17]-[25]). Большинство из этих ядер могут быть представлены в виде

$$
V(\lambda, \mu)=\frac{\sqrt{F(\lambda) F(\mu)}}{2 i \pi(\lambda-\mu)}\left[e_{+}(\lambda) e_{-}(\mu)-e_{-}(\lambda) e_{+}(\mu)\right]
$$

где

$$
e_{ \pm}(\lambda)=\exp \left( \pm \frac{i x p(\lambda)}{2} \pm \frac{g(\lambda)}{2}\right)
$$

а оператор $I+\gamma V$ действует на некотором контуре $\mathcal{C}$. Мы называем ядро (1.2) обобщенным синус-ядром (ОСЯ). В упомянутых выше работах исследовалось асимптотическое поведение детерминанта Фредгольма $\operatorname{det}_{\mathcal{C}}(I+\gamma V)$ при большом $x$ для некоторых частных случаев функций $F, p, g$ и контура $\mathcal{C}$. В работе [25] было показано, что асимптотическое разложение детерминанта Фредгольма зависит только от аналитических свойств функций $F, p$ и $g$ вблизи контура $\mathcal{C}$. Зная эти свойства, можно вычислить асимптотическое поведение, не используя явный вид функций.

В настоящей статье мы сосредоточим наше внимание на частном случае ОСЯ, когда интегральный оператор $I+\gamma V$ действует на всей вещественной оси. Мы также полагаем $p(\lambda)=\lambda$, поскольку именно эта ситуация возникает в большинстве приложений. Данный случай проще случая конечного интервала (и тем более объединения конечных интервалов). Дело в том, что для конечного $\ell$ асимптотическое поведение $\operatorname{det}_{\ell}(I+\gamma V)$ при большом $x$ в основном следует из аналитических свойств резольвенты оператора $I+\gamma V$ в окрестностях концов интервала $\ell$. Несмотря на то что методы подобного анализа в настоящее время хорошо развиты (см. статьи [12], [25]), технически они являются довольно сложными. В результате даже в простейших случаях обычно удается явно получить лишь несколько первых членов асимптотического разложения.

В случае операторов, действующих на $\mathbb{R}$, указанная трудность исчезает в силу отсутствия концов интервала. Благодаря этому можно получить равномерные оценки для резольвенты на всей вещественной оси с точностью до экспоненциально малых поправок. В результате можно продвинуться гораздо дальше в изучении асимптотического поведения детерминанта Фредгольма. В некоторых частных случаях удается получить даже полное асимптотическое разложение [20].

2 Теоретическая и математическая физика, т. 165, № 1, 2010 г. 


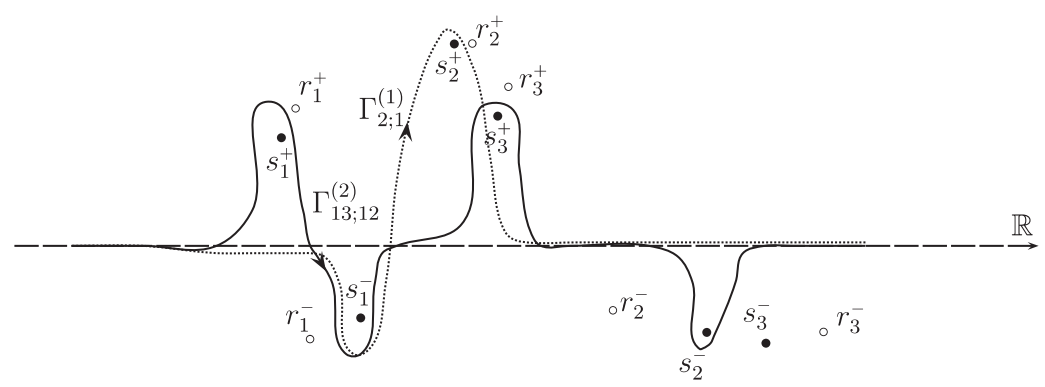

Рис. 1. Полюсы $r_{j}^{ \pm}$показаны как о, нули $s_{j}^{ \pm}$уравнения $1+\gamma F(\lambda)=0$ показаны как •. Контур интегрирования $\Gamma_{13 ; 12}^{(2)}$ (сплошная линия) обходит точки $s_{1}^{+}, s_{3}^{+}$сверху, а точки $s_{1}^{-}, s_{2}^{-}-$снизу. Контур $\Gamma_{2 ; 1}^{(1)}$ (пунктирная линия) обходит точку $s_{2}^{+}$сверху, а $s_{1}^{-}-$снизу.

Аналогичная картина возникает в случае ОСЯ, действующего на замкнутом контуре $\mathcal{C}$. Для таких ядер функция $p(\lambda)$ обычно такова, что $\left|e^{i x p(\lambda)}\right|=1$ при $\lambda \in \mathcal{C}$. Методы асимптотического анализа, предложенные в настоящей статье, также могут быть использованы для этого случая.

Статья построена следующим образом. В разделе 2 мы анонсируем основные результаты работы, а именно асимптотическую формулу для резольвенты оператора $I+\gamma V$ и два вида асимптотического разложения $\operatorname{det}_{\mathbb{R}}(I+\gamma V)$. Доказательства этих теорем даны в разделах 3-5. В разделе 6 рассмотрены два приложения полученных результатов.

\section{2. ОБОЗНАЧЕНИЯ И ОСНОВНЫЕ ТЕОРЕМЫ}

В этом разделе мы конкретизируем некоторые свойства функций $g(\lambda)$ и $F(\lambda)$, входящих в ядро (1.2). Напомним, что мы положили $p(\lambda)=\lambda$.

Пусть функция $g(\lambda)$ голоморфна в полосе $|\operatorname{Im} \lambda|<a, a>0$. Предположим также, что $g(\lambda)$ остается ограниченной при $\operatorname{Re} \lambda \rightarrow \pm \infty$ и $|\operatorname{Im} \lambda|<a$. Функция $F(\lambda)$ голоморфна в той же полосе за исключением $n_{+}+n_{-}$простых полюсов в точках $r_{j}^{ \pm}$, отделенных от вещественной оси ${ }^{1)}$. Мы считаем, что точки $r_{j}^{+}, j=$ $1, \ldots, n_{+}$, лежат в верхней полуплоскости, а точки $r_{k}^{-}, k=1, \ldots, n_{-}$, лежат в нижней полуплоскости (см. рис. 1). Комплексное число $\gamma$ предполагается достаточно малым, так что нули $s_{j}^{ \pm}$функции $1+\gamma F(\lambda)$ слегка сдвинуты по отношению к полюсам $r_{j}^{ \pm}$ (см. рис. 1). Нули $s_{j}^{+}, j=1, \ldots, n_{+}$, принадлежат верхней полуплоскости, а $s_{k}^{-}$, $k=1, \ldots, n_{-}$, принадлежат нижней полуплоскости. Наконец, мы предполагаем, что $F(\lambda) \rightarrow 0$ при $\operatorname{Re} \lambda \rightarrow \pm \infty,|\operatorname{Im} \lambda|<a$ таким образом, что $|\operatorname{tr} V|<\infty$.

При условиях, перечисленных выше, детерминант $Ф$ редгольма $\operatorname{det}_{\mathbb{R}}(I+\gamma V)$ существует и является целой функцией от $\gamma$. Так как при $\gamma=0$ этот детерминант равен 1 ,

${ }^{1)}$ Случай полюсов более высокого порядка можно проанализировать, беря пределы $r_{j}^{ \pm} \rightarrow r_{k}^{ \pm}$. Мы не рассматриваем этот случай, поскольку тогда явные формулы становятся гораздо более громоздкими. 
мы заключаем, что $\operatorname{det}_{\mathbb{R}}(I+\gamma V) \neq 0$, если $\gamma$ принадлежит некоторой окрестности нуля. Следовательно, резольвента оператора $I+\gamma V$ существует по крайней мере для достаточно малых $\gamma$.

Введем теперь несколько функций, которые будут использоваться ниже. Прежде всего, определим функции $\nu(\lambda)$ и $\alpha(\lambda)$ как

$$
\nu(\lambda)=-\frac{1}{2 \pi i} \ln (1+\gamma F(\lambda)), \quad \alpha(\lambda) \equiv \alpha([\nu], \lambda)=\exp \left(\int_{\mathbb{R}} \frac{\nu(\mu) d \mu}{\mu-\lambda}\right) .
$$

В формуле (2.1) ветвь логарифма фиксирована условием $\nu(+\infty)=0$. Ясно, что функция $\alpha(\lambda)$ имеет разрез на вещественной оси, причем ее предельные значения $\alpha_{ \pm}(\lambda)$ из верхней и нижней полуплоскостей удовлетворяют свойству

$$
\alpha_{-}(\lambda)=\alpha_{+}(\lambda)(1+\gamma F(\lambda)), \quad \lambda \in \mathbb{R} .
$$

Предельное значение $\alpha_{+}(\lambda)$ (предельное значение $\alpha_{-}(\lambda)$ ) может быть аналитически продолжено в верхнюю (соответственно в нижнюю) полуплоскость, где оно не обращается в нуль. При $\lambda \rightarrow \infty$ функция $\alpha(\lambda)$ ведет себя как

$$
\alpha(\lambda)=1+\frac{\alpha_{1}}{\lambda}+O\left(\lambda^{-2}\right), \quad \text { где } \quad \alpha_{1}=-\int_{\mathbb{R}} \nu(\mu) d \mu .
$$

Определим также $\left(n_{+} \times n_{-}\right)$-матрицу $A^{-}$и $\left(n_{-} \times n_{+}\right)$-матрицу $A^{+}$:

$$
A_{j k}^{-}=\frac{h_{k}^{-} e_{-}^{2}\left(s_{k}^{-}\right)}{s_{j}^{+}-s_{k}^{-}}, \quad A_{j k}^{+}=\frac{h_{k}^{+} e_{+}^{2}\left(s_{k}^{+}\right)}{s_{j}^{-}-s_{k}^{+}}, \quad \text { где } \quad h_{k}^{ \pm}=-\frac{\left(\alpha_{ \pm}\left(s_{k}^{ \pm}\right)\right)^{\mp 2}}{\gamma F^{\prime}\left(s_{k}^{ \pm}\right)} .
$$

Введем также набор контуров $\Gamma_{J ; K}^{(n)}$ с $n=0,1, \ldots, \min \left(n_{+}, n_{-}\right)$. Здесь $J$ и $K$ суть мультииндексы: $J=\left\{j_{1}, \ldots, j_{n}\right\}$ с $1 \leqslant j_{s} \leqslant n_{+}$и $K=\left\{k_{1}, \ldots, k_{n}\right\}$ с $1 \leqslant k_{s} \leqslant n_{-}$. По определению мы полагаем $\Gamma^{(0)}=\mathbb{R}$. Контур $\Gamma_{J ; K}^{(n)}$ является такой деформацией вещественной оси, что, двигая $\mathbb{R}$ к $\Gamma_{J ; K}^{(n)}$, мы пересекаем только корни $s_{j_{1}}^{+}, \ldots, s_{j_{n}}^{+}$и $s_{k_{1}}^{-}, \ldots, s_{k_{n}}^{-}$, в то время как остальные корни $s_{\ell}^{ \pm}$и все полюсы $r_{\ell}^{ \pm}$пересекаться не должны (см. рис. 1).

Наконец, пусть

$$
\mathcal{A}_{\mathcal{C}}([g],[\nu])=-\int_{\mathcal{C}}\left(i x+g^{\prime}(\lambda)\right) \nu(\lambda) d \lambda+\iint_{\mathcal{C}} \frac{\nu(\lambda) \nu(\mu)}{\left(\lambda-\mu_{+}\right)^{2}} d \lambda d \mu .
$$

Здесь контур $\mathcal{C}$ является одним из контуров $\Gamma_{J ; K}^{(n)}$. Символ $\mu_{+}$означает, что переменная $\mu$ слегка сдвинута с контура интегрирования $\mathcal{C}$ влево. Отметим, что разрезы функции $\nu(\lambda)$ можно всегда выбрать так, что контур $\mathcal{C}$ не пересекает их.

Теперь мы готовы к тому, чтобы сформулировать основные теоремы об асимптотическом поведении резольвенты и $\operatorname{det}_{\mathbb{R}}(I+\gamma V)$.

TeOPEMA 2.1. Пусть $x \rightarrow \infty u$

$$
R(\lambda, \mu)+\gamma \int_{\mathbb{R}} V(\lambda, \xi) R(\xi, \mu) d \xi=V(\lambda, \mu) .
$$


Тогда $R(\lambda, \mu)$ имеет следующий вид:

$$
R(\lambda, \mu)=\frac{\sqrt{F(\lambda) F(\mu)}}{2 i \pi(\lambda-\mu)}\left[f_{+}(\lambda) f_{-}(\mu)-f_{-}(\lambda) f_{+}(\mu)\right],
$$

где

$$
\begin{aligned}
f_{ \pm}(\lambda)= & \alpha_{\mp}^{\mp 1}(\lambda) e_{ \pm}(\lambda)\left[1+\sum_{j=1}^{n_{\mp}} \frac{D_{j}^{ \pm} h_{j}^{\mp} e_{\mp}^{2}\left(s_{j}^{\mp}\right)}{\lambda-s_{j}^{\mp}}\right]+ \\
& +\alpha_{ \pm}^{ \pm 1}(\lambda) e_{\mp}(\lambda) \sum_{j=1}^{n_{ \pm}} \frac{C_{j}^{ \pm} h_{j}^{ \pm} e_{ \pm}^{2}\left(s_{j}^{ \pm}\right)}{\lambda-s_{j}^{ \pm}}+O\left(e^{-a x}\right)
\end{aligned}
$$

равномерно по $\lambda \in \mathbb{R}$. Константы $C_{j}^{ \pm}$и $D_{j}^{ \pm}$могут быть найденъ из систем уравнений

$$
\begin{array}{ll}
C_{j}^{+}-\sum_{k=1}^{n_{+}} A_{j k} C_{k}^{+}=1, & C_{j}^{-}-\sum_{k=1}^{n_{-}} \tilde{A}_{j k} C_{k}^{-}=1, \\
D_{j}^{+}-\sum_{k=1}^{n_{+}} A_{j k}^{+} C_{k}^{+}=0, & D_{j}^{-}-\sum_{k=1}^{n_{-}} A_{j k}^{-} C_{k}^{-}=0,
\end{array}
$$

где $A=A^{-} A^{+}$и $\tilde{A}=A^{+} A^{-}$.

Эта теорема доказана в следующем разделе.

ЗАмечАниЕ. При достаточно большом $x$ элементы матриц $A^{ \pm}$экспоненциально малы. Поэтому $\operatorname{det}(I-A) \neq 0$ и $\operatorname{det}(I-\tilde{A}) \neq 0$, а следовательно, каждая из систем (2.9) имеет единственное решение.

Теорема 2.2. Пусть $x \rightarrow \infty$. Тогда детерминант Фредголъма оператора $I+\gamma V$ ведет себя как

$$
\operatorname{det}_{\mathbb{R}}(I+\gamma V)=e^{\mathcal{A}_{\mathbb{R}}([g],[\nu])} \operatorname{det}_{n_{+}}(I-A)\left(1+O\left(e^{-a x}\right)\right) .
$$

Здесъ функиионал $\mathcal{A}_{\mathbb{R}}([g],[\nu])$ дается формулой $(2.5)$ с $\mathcal{C}=\mathbb{R}$.

Доказательство этой теоремы дано в разделе 4.

ЗАмЕчАниЕ. В формуле (2.10) определитель $\left(n_{+} \times n_{+}\right)$-матрицы $I-A$ может быть заменен на определитель $\left(n_{-} \times n_{-}\right)$-матрицы $I-\tilde{A}$.

Теорема 2.3. Пусть $x \rightarrow \infty$. Тогда детерминант Фредголъма оператора $I+\gamma V$ ведет себя как

$$
\operatorname{det}_{\mathbb{R}}(I+\gamma V)=\sum_{\mathcal{C}} e^{\mathcal{A}_{\mathcal{C}}([g],[\nu])}\left(1+O\left(e^{-a x}\right)\right),
$$

где функиионал $\mathcal{A}_{\mathcal{C}}([g],[\nu])$ задан формулой $(2.5)$, а сумма берется по всем возможным контурам $\mathcal{C} \in\left\{\Gamma_{J ; K}^{(n)}\right\}$ включая $\Gamma^{(0)}=\mathbb{R}$.

Доказательство этой теоремы дано в разделе 5. 


\section{3. АСИМПТОТИЧЕСКАЯ ФОРМУЛА ДЛЯ РЕЗОЛЬВЕНТЫ}

Этот раздел посвящен доказательству теоремы 2.1. Оператор $I+\gamma V$ принадлежит классу вполне интегрируемых операторов [24], [27]. Известно [19], [24], [27], что для таких операторов ядро резольвенты имеет вид $(2.7)$, где функции $f_{ \pm}(\lambda)$ удовлетворяют интегральному уравнению

$$
f_{ \pm}(\lambda)+\frac{\gamma}{2 \pi i} \int_{\mathbb{R}} \frac{e_{+}(\lambda) e_{-}(\mu)-e_{-}(\lambda) e_{+}(\mu)}{\lambda-\mu} F(\mu) f_{ \pm}(\mu) d \mu=e_{ \pm}(\lambda) .
$$

Таким образом, для доказательства теоремы 2.1 нам следует асимптотически решить уравнение (3.1) с точностью до членов порядка $O\left(e^{-a x}\right)$.

ДоКАЗАТЕЛЬСТво тЕОРЕмЫ 2.1. Поскольку $\operatorname{det}_{\mathbb{R}}(I+\gamma V) \neq 0$ при достаточно малом $\gamma$, решение уравнения (3.1) существует и единственно. Поэтому нам достаточно подставить формулы (2.8) в интегральное уравнение (3.1) и проверить, что последнее выполняется с точностью до членов порядка $O\left(e^{-a x}\right)$.

Рассмотрим, например, уравнение для $f_{+}(\lambda)$. Совершая подстановку, находим

$$
\begin{aligned}
f_{+}(\lambda)-e_{+}(\lambda)= & \frac{1}{2 \pi i} \int_{\mathbb{R}} \frac{\gamma F(\mu) \alpha_{-}^{-1}(\mu) d \mu}{\mu-\lambda+i 0} \times \\
& \times\left[1+\sum_{j=1}^{n_{-}} \frac{D_{j}^{+} h_{j}^{-} e_{-}^{2}\left(s_{j}^{-}\right)}{\mu-s_{j}^{-}}\right]\left(e_{+}(\lambda)-e_{-}(\lambda) e_{+}^{2}(\mu)\right)+ \\
& +\frac{1}{2 \pi i} \int_{\mathbb{R}} \frac{\gamma F(\mu) \alpha_{+}(\mu) d \mu}{\mu-\lambda-i 0} \sum_{j=1}^{n_{+}} \frac{C_{j}^{+} h_{j}^{+} e_{+}^{2}\left(s_{j}^{+}\right)}{\mu-s_{j}^{+}} \times \\
& \times\left(e_{+}(\lambda) e_{-}^{2}(\mu)-e_{-}(\lambda)\right)+O\left(e^{-a x}\right) .
\end{aligned}
$$

Для удобства мы сдвинули $\mu-\lambda$ на $+i 0$ в первом интеграле и на $-i 0$ во втором. Рассмотрим коэффициент при $e_{+}(\lambda)$. Имеем

$$
\begin{aligned}
\alpha_{-}^{-1}(\lambda) & {\left[1+\sum_{j=1}^{n_{-}} \frac{D_{j}^{+} h_{j}^{-} e_{-}^{2}\left(s_{j}^{-}\right)}{\lambda-s_{j}^{-}}\right]-1=} \\
= & \frac{1}{2 \pi i} \int_{\mathbb{R}} \frac{\gamma F(\mu) \alpha_{-}^{-1}(\mu) d \mu}{\mu-\lambda+i 0}\left[1+\sum_{j=1}^{n_{-}} \frac{D_{j}^{+} h_{j}^{-} e_{-}^{2}\left(s_{j}^{-}\right)}{\mu-s_{j}^{-}}\right]+ \\
& +\frac{1}{2 \pi i} \int_{\mathbb{R}} \frac{\gamma F(\mu) \alpha_{+}(\mu) e_{-}^{2}(\mu) d \mu}{\mu-\lambda-i 0} \sum_{j=1}^{n_{+}} \frac{C_{j}^{+} h_{j}^{+} e_{+}^{2}\left(s_{j}^{+}\right)}{\mu-s_{j}^{+}}+O\left(e^{-a x}\right) .
\end{aligned}
$$

Первый интеграл в правой части уравнения (3.3) может быть взят в явном виде с помощью соотношения $\gamma F(\lambda) \alpha_{-}^{-1}(\lambda)=\alpha_{+}^{-1}(\lambda)-\alpha_{-}^{-1}(\lambda)$. Функции $\alpha_{ \pm}(\lambda)$ аналитичны и не равны нулю в соответствующих полуплоскостях, таким образом, благодаря условию (2.3) мы получаем

$$
\frac{1}{2 \pi i} \int_{\mathbb{R}} \frac{\gamma F(\mu) \alpha_{-}^{-1}(\mu) d \mu}{\mu-\lambda+i 0}\left[1+\sum_{j=1}^{n_{-}} \frac{D_{j}^{+} h_{j}^{-} e_{-}^{2}\left(s_{j}^{-}\right)}{\mu-s_{j}^{-}}\right]=
$$




$$
\begin{aligned}
= & \frac{1}{2 \pi i} \int_{\mathbb{R}} d \mu \frac{\alpha_{+}^{-1}(\mu)-1-\left(\alpha_{-}^{-1}(\mu)-1\right)}{\mu-\lambda+i 0}+ \\
& +\frac{1}{2 \pi i} \int_{\mathbb{R}} d \mu \frac{\alpha_{+}^{-1}(\mu)-\alpha_{-}^{-1}(\mu)}{\mu-\lambda+i 0} \sum_{j=1}^{n_{-}} \frac{D_{j}^{+} h_{j}^{-} e_{-}^{2}\left(s_{j}^{-}\right)}{\mu-s_{j}^{-}}= \\
= & \alpha_{-}^{-1}(\lambda)\left[1+\sum_{j=1}^{n_{-}} \frac{D_{j}^{+} h_{j}^{-} e_{-}^{2}\left(s_{j}^{-}\right)}{\lambda-s_{j}^{-}}\right]-1-\sum_{j=1}^{n_{-}} \frac{\alpha_{-}^{-1}\left(s_{j}^{-}\right) D_{j}^{+} h_{j}^{-} e_{-}^{2}\left(s_{j}^{-}\right)}{\lambda-s_{j}^{-}} .
\end{aligned}
$$

Следовательно, уравнение (3.3) принимает вид

$$
\begin{aligned}
& \sum_{j=1}^{n_{-}} \frac{\alpha_{-}^{-1}\left(s_{j}^{-}\right) D_{j}^{+} h_{j}^{-} e_{-}^{2}\left(s_{j}^{-}\right)}{\lambda-s_{j}^{-}}= \\
& \quad=\frac{1}{2 \pi i} \int_{\mathbb{R}} \frac{\gamma F(\mu) \alpha_{-}(\mu) e_{-}^{2}(\mu) d \mu}{(1+\gamma F(\mu))(\mu-\lambda-i 0)} \sum_{k=1}^{n_{+}} \frac{C_{k}^{+} h_{k}^{+} e_{+}^{2}\left(s_{k}^{+}\right)}{\mu-s_{k}^{+}}+O\left(e^{-a x}\right) .
\end{aligned}
$$

Здесь мы воспользовались тем, что $\alpha_{+}(\mu)=\alpha_{-}(\mu)(1+\gamma F(\mu))^{-1}$. Оставшийся интеграл может быть вычислен асимптотически с помощью вычетов в точках $\mu$ в нижней полуплоскости, где $1+\gamma F(\mu)=0$. Действительно, контур интегрирования в формуле (3.5) можно сдвинуть в нижнюю полуплоскость по крайней мере вплоть до $\operatorname{Im} \mu=-a$. Тогда подынтегральное выражение становится пропорциональным $e^{-a x}$. Следовательно, с точностью до поправок порядка $O\left(e^{-a x}\right)$ интеграл в формуле (3.5) равен сумме вычетов в точках $s_{j}^{-}$. Сравнивая коэффициенты при всех $\left(\lambda-s_{j}^{-}\right)^{-1}$, получаем

$$
D_{j}^{+}=\sum_{k=1}^{n_{+}} A_{j k}^{+} C_{k}^{+} .
$$

Аналогичное вычисление для коэффициента при $e_{-}(\lambda)$ в формуле (3.2) приводит к уравнениям

$$
C_{j}^{+}=1+\sum_{k=1}^{n_{-}} A_{j k}^{-} D_{k}^{+} .
$$

Уравнения (3.6), (3.7) немедленно влекут первую из систем (2.9). Вторая система (2.9) следует из анализа интегрального уравнения для функции $f_{-}(\lambda)$, который может быть проведен тем же методом.

\section{4. ПЕРВАЯ АСИМПТОТИЧЕСКАЯ ФОРМУЛА ДЛЯ ДЕТЕРМИНАНТА}

В работе [26] были получены главные члены асимптотического разложения детерминанта Фредгольма $\operatorname{det}_{\mathbb{R}}(I+\gamma V)$ (см. также [28] для случая $\left.g(\lambda)=0\right)$ :

$$
\ln \operatorname{det}_{\mathbb{R}}(I+\gamma V)=\mathcal{A}_{\mathbb{R}}([g],[\nu])+o(1), \quad x \rightarrow \infty .
$$

Таким образом, нам нужно доказать только то, что поправки к этой формуле имеют вид определителя $\operatorname{det}(I-A)$ матрицы конечного порядка. 
ДокАЗАТЕЛЬСТво тЕОРЕмы 2.2. Поправки к формуле (4.1) могут быть вычислены из следующего тождества [19], [24]:

$$
\partial_{x} \ln \operatorname{det}_{\mathbb{R}}(I+\gamma V)=\frac{\gamma}{2 \pi} \int_{\mathbb{R}} f_{+}(\lambda) e_{-}(\lambda) F(\lambda) d \lambda .
$$

Подставляя сюда выражение $(2.8)$ для $f_{+}(\lambda)$, мы получаем

$$
\begin{aligned}
\partial_{x} \ln \operatorname{det}(I+\gamma V)= & \frac{1}{2 \pi} \int_{\mathbb{R}} \gamma F(\lambda) \alpha_{-}^{-1}(\lambda)\left[1+\sum_{j=1}^{n_{-}} \frac{D_{j}^{+} h_{j}^{-} e_{-}^{2}\left(s_{j}^{-}\right)}{\lambda-s_{j}^{-}}\right] d \lambda+ \\
& +\frac{1}{2 \pi} \int_{\mathbb{R}} \gamma F(\lambda) \alpha_{+}(\lambda) e_{-}^{2}(\lambda) \sum_{j=1}^{n_{+}} \frac{C_{j}^{+} h_{j}^{+} e_{+}^{2}\left(s_{j}^{+}\right)}{\lambda-s_{j}^{+}}+O\left(e^{-a x}\right) .
\end{aligned}
$$

Дадим несколько комментариев по поводу вычисления интеграла

$$
\frac{1}{2 \pi} \int_{\mathbb{R}} \gamma F(\lambda) \alpha_{-}^{-1}(\lambda) d \lambda .
$$

Имеем

$$
\begin{aligned}
\gamma F(\lambda) \alpha_{-}^{-1}(\lambda)= & \alpha_{+}^{-1}(\lambda)-\alpha_{-}^{-1}(\lambda)=\left(\alpha_{+}^{-1}(\lambda)-1+\frac{\alpha_{1}}{\lambda-\lambda_{0}}\right)- \\
& -\left(\alpha_{-}^{-1}(\lambda)-1+\frac{\alpha_{1}}{\lambda-\lambda_{0}}\right)
\end{aligned}
$$

где $\lambda_{0}$ - произвольное комплексное число с положительной мнимой частью. Заметим, что в силу (2.3) оба члена в правой части (4.5) ведут себя как $O\left(\lambda^{-2}\right)$ при $\lambda \rightarrow \infty$. Поэтому эти слагаемые можно интегрировать по отдельности. Интеграл от второго члена равен нулю, так как подынтегральное выражение аналитично в нижней полуплоскости. Первое слагаемое в (4.5) имеет только один простой полюс в верхней полуплоскости, поэтому

$$
\frac{1}{2 \pi} \int_{\mathbb{R}} \gamma F(\lambda) \alpha_{-}^{-1}(\lambda) d \lambda=\frac{1}{2 \pi} \int_{\mathbb{R}}\left(\alpha_{+}^{-1}(\lambda)-1+\frac{\alpha_{1}}{\lambda-\lambda_{0}}\right) d \lambda=i \alpha_{1} .
$$

Другие интегралы в (4.3) могут быть вычислены аналогично интегралам, рассмотренным в предыдущем разделе. А именно, интеграл в первой строке формулы (4.3) может быть взят явно, аналогично интегралу (3.4). Интеграл во второй строке формулы (4.3) может быть вычислен асимптотически так же, как интеграл (3.5). Воспользовавшись затем уравнениями (2.9), мы после несложных преобразований получаем

$$
\partial_{x} \ln \operatorname{det}(I+\gamma V)=i \alpha_{1}-\sum_{j=1}^{n_{+}} A_{j j}^{\prime} C_{j}^{+}+O\left(e^{-a x}\right)
$$

где штрих означает производную по $x$. Используя явное выражение (2.4) для $A$, легко убедиться в том, что

$$
A_{j j}^{\prime}=A_{\ell j}^{\prime}+i\left(s_{\ell}^{+}-s_{j}^{+}\right) A_{\ell j} .
$$


Тогда в силу (2.9) имеем

$$
\begin{gathered}
\partial_{x} \ln \operatorname{det}(I+\gamma V)-i \alpha_{1}=-\sum_{j, \ell=1}^{n_{+}}\left[A_{\ell j}^{\prime}+i\left(s_{\ell}^{+}-s_{j}^{+}\right) A_{\ell j}\right](I-A)_{j \ell}^{-1}+O\left(e^{-a x}\right)= \\
=\partial_{x} \operatorname{tr} \ln (I-A)+i \sum_{j, \ell=1}^{n_{+}}\left(s_{\ell}^{+}-s_{j}^{+}\right)\left(\delta_{\ell j}-A_{\ell j}\right)(I-A)_{j \ell}^{-1}+O\left(e^{-a x}\right)= \\
=\partial_{x} \ln \operatorname{det}(I-A)-i \sum_{j=1}^{n_{+}} s_{j}^{+}+i \sum_{\ell=1}^{n_{+}} s_{\ell}^{+}+O\left(e^{-a x}\right)= \\
=\partial_{x} \ln \operatorname{det}(I-A)+O\left(e^{-a x}\right) .
\end{gathered}
$$

Интегрируя по $x$, мы приходим к соотношению

$$
\ln \operatorname{det}(I+\gamma V)=i x \alpha_{1}+C+\ln \operatorname{det}(I-A)+O\left(e^{-a x}\right),
$$

сравнивая его с (4.1), находим константу интегрирования $C$ :

$$
C=-\int_{\mathbb{R}} g^{\prime}(\lambda) \nu(\lambda) d \lambda+\iint_{\mathbb{R}} \frac{\nu(\lambda) \nu(\mu)}{\left(\lambda-\mu_{+}\right)^{2}} d \lambda d \mu=\left.\mathcal{A}_{\mathbb{R}}([g],[\nu])\right|_{x=0} .
$$

Таким образом, мы нашли поправки к формуле (4.1), и нам остается только доказать, что определитель $\left(n_{+} \times n_{+}\right)$-матрицы $I-A$ равен определителю $\left(n_{-} \times n_{-}\right)$-матрицы $I-\tilde{A}$. Для этого достаточно представить $\operatorname{det}(I-A)$ в виде определителя блочной матрицы размера $\left(n_{+}+n_{-}\right) \times\left(n_{+}+n_{-}\right)$:

$$
\operatorname{det}(I-A)=\operatorname{det}\left(I-A^{-} A^{+}\right)=\operatorname{det}\left(\begin{array}{cc}
I & A^{+} \\
A^{-} & I
\end{array}\right)=\operatorname{det}\left(I-A^{+} A^{-}\right)=\operatorname{det}(I-\tilde{A}),
$$

что завершает доказательство.

\section{5. ВТОРАЯ АСИМПТОТИЧЕСКАЯ ФОРМУЛА ДЛЯ ДЕТЕРМИНАНТА}

Определим набор функций, аналогичных функции $\alpha(\lambda)(2.1)$ :

$$
\alpha\left(\lambda ; \Gamma_{J ; K}^{(n)}\right)=\exp \left(\int_{\Gamma_{J ; K}^{(n)}} \frac{\nu(\mu) d \mu}{\mu-\lambda}\right),
$$

где $\nu(\lambda)$ по-прежнему дается формулой (2.1). Очевидно,

$$
1+\gamma F(\lambda)=\frac{\alpha_{-}\left(\lambda ; \Gamma_{J ; K}^{(n)}\right)}{\alpha_{+}\left(\lambda ; \Gamma_{J ; K}^{(n)}\right)}, \quad \lambda \in \Gamma_{J ; K}^{(n)} .
$$

Здесь функция $\alpha_{+}$(функция $\alpha_{-}$) есть предельное значение функции $\alpha\left(\lambda ; \Gamma_{J ; K}^{(n)}\right)$ на контуре $\Gamma_{J ; K}^{(n)}$ слева (соответственно справа). Функция $\alpha_{-}$аналитична и не обращается в нуль в области справа от $\Gamma_{J ; K}^{(n)}$, она имеет нули в точках $\lambda=s_{\ell}^{+}, \ell \notin J$ и полюсы в точках $\lambda=r_{\ell}^{+}, \ell=1, \ldots, n_{+}$. Аналогично функция $\alpha_{+}^{-1}$ аналитична и не обращается в нуль в области слева от $\Gamma_{J ; K}^{(n)}$, она имеет нули в точках $\lambda=s_{\ell}^{-}, \ell \notin K$, и полюсы в точках $\lambda=r_{\ell}^{-}, \ell=1, \ldots, n_{-}$. 


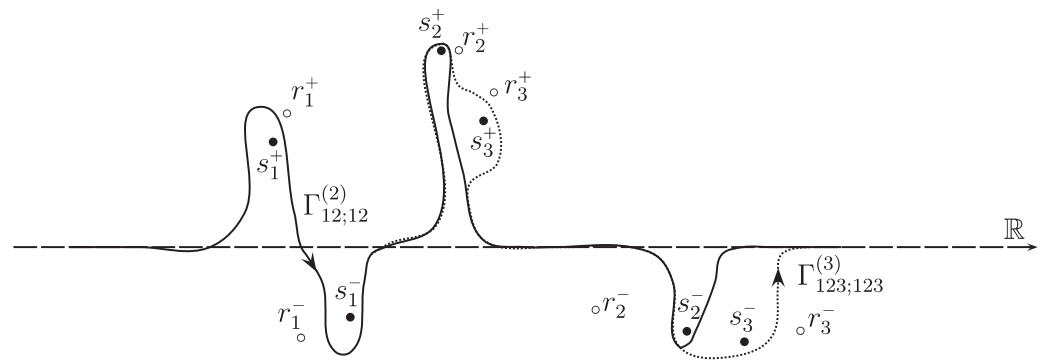

Рис. 2. Контуры интегрирования $\Gamma_{12 ; 12}^{(2)}$ (сплошная линия) и $\Gamma_{123 ; 123}^{(3)}$ (пунктирная линия).

Лемма. Пусть $\nu(\lambda)$ дана формулой (2.1). Тогда

$$
\begin{aligned}
\exp \{ & \left.\mathcal{A}_{\Gamma_{J ; K}^{(n)}}([g],[\nu])-\mathcal{A}_{\Gamma_{\hat{J} ; \hat{K}}^{(n-1)}}([g],[\nu])\right\}= \\
& =\frac{\alpha_{-}^{2}\left(s_{n}^{-} ; \Gamma_{\hat{J} ; \hat{K}}^{(n-1)}\right) \alpha_{+}^{-2}\left(s_{n}^{+} ; \Gamma_{\hat{J} ; \hat{K}}^{(n-1)}\right)}{\left(s_{j_{n}}^{+}-s_{k_{n}}^{-}\right)^{2} \gamma^{2} F^{\prime}\left(s_{j_{n}}^{+}\right) F^{\prime}\left(s_{k_{n}}^{-}\right)} e_{+}^{2}\left(s_{j_{n}}^{+}\right) e_{-}^{2}\left(s_{k_{n}}^{-}\right),
\end{aligned}
$$

где $\hat{J}=J \backslash j_{n} u \hat{K}=K \backslash k_{n}$.

ДоказАтельство. Рассмотрим первое слагаемое в $(2.5)$, полагая $\mathcal{C}=\Gamma_{J ; K}^{(n)}$ и $\mathcal{C}=\Gamma_{\hat{J} ; \hat{K}}^{(n-1)}$. Контур $\Gamma_{J ; K}^{(n)}$ получен деформацией контура $\Gamma_{\hat{J} ; \hat{K}}^{(n-1)}$, при которой пересекаются корни уравнения $1+\gamma F(\lambda)$ в точках $s_{j_{n}}^{+}$и $s_{k_{n}}^{-}$(см. рис. 2). Поэтому имеем

$$
-\left(\int_{\Gamma_{J ; K}^{(n)}}^{(n)}-\int_{\Gamma_{\hat{J} ; \hat{K}}^{(n-1)}}\right)\left(i x+g^{\prime}(\lambda)\right) \nu(\lambda) d \lambda=\int_{s_{k_{n}}^{-}}^{s_{j_{n}}^{+}}\left(i x+g^{\prime}(\lambda)\right) d \lambda=\ln \left(e_{+}^{2}\left(s_{j_{n}}^{+}\right) e_{-}^{2}\left(s_{k_{n}}^{-}\right)\right) .
$$

Преобразуем теперь двойной интеграл в (2.5). Для облегчения обозначений положим

$$
\alpha\left(\lambda ; \Gamma_{J ; K}^{(n)}\right)=\alpha(\lambda ; n), \quad \alpha\left(\lambda ; \Gamma_{\hat{J} ; \hat{K}}^{(n-1)}\right)=\alpha(\lambda ; n-1) .
$$

На контуре $\Gamma_{\hat{J} ; \hat{K}}^{(n-1)}$ функция $\nu(\lambda)$ может быть представлена в виде

$$
\nu(\lambda)=-\frac{1}{2 \pi i} \ln \frac{\alpha_{-}(\lambda ; n-1)}{\alpha_{+}(\lambda ; n-1)}, \quad \lambda \in \Gamma_{\hat{J} ; \hat{K}}^{(n-1)} .
$$

Воспользовавшись этим представлением, можно проинтегрировать по $\lambda$ в двойном интеграле в (2.5):

$$
\iint_{\Gamma_{\hat{J} ; \hat{K}}^{(n-1)}} \frac{\nu(\lambda) \nu(\mu)}{\left(\lambda-\mu_{+}\right)^{2}} d \lambda d \mu=\int_{\Gamma_{\hat{J} ; \hat{K}}^{(n-1)}} \nu(\mu) \cdot \ln ^{\prime} \alpha_{+}(\mu ; n-1) d \mu
$$


Подставляя сюда $\nu(\mu)$ в виде (5.6), мы приходим к

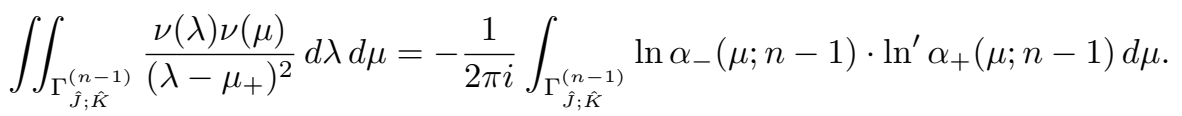

Аналогично на контуре $\Gamma_{J ; K}^{(n)}$ имеем

$$
\iint_{\Gamma_{J ; K}^{(n)}} \frac{\nu(\lambda) \nu(\mu)}{\left(\lambda-\mu_{+}\right)^{2}} d \lambda d \mu=-\frac{1}{2 \pi i} \int_{\Gamma_{J ; K}^{(n)}} \ln \alpha_{-}(\mu ; n) \cdot \ln ^{\prime} \alpha_{+}(\mu ; n) d \mu .
$$

Для того чтобы сравнить (5.8) и (5.9), мы сначала заметим, что

$$
\alpha_{ \pm}(\lambda ; n)=\alpha_{ \pm}(\lambda ; n-1)\left(\frac{\lambda-s_{k_{n}}^{-}}{\lambda-s_{j_{n}}^{+}}\right) .
$$

Введем теперь функции $\hat{\alpha}_{ \pm}(\lambda)$ с помощью равенств

$$
\alpha_{-}(\lambda ; n-1)=\hat{\alpha}_{-}(\lambda)\left(\frac{\lambda-s_{j_{n}}^{+}}{\lambda-r_{j_{n}}^{+}}\right), \quad \alpha_{+}(\lambda ; n-1)=\hat{\alpha}_{+}(\lambda)\left(\frac{\lambda-r_{k_{n}}^{-}}{\lambda-s_{k_{n}}^{-}}\right) .
$$

В силу (5.10) мы также имеем

$$
\alpha_{-}(\lambda ; n)=\hat{\alpha}_{-}(\lambda)\left(\frac{\lambda-s_{k_{n}}^{-}}{\lambda-r_{j_{n}}^{+}}\right), \quad \alpha_{+}(\lambda ; n)=\hat{\alpha}_{+}(\lambda)\left(\frac{\lambda-r_{k_{n}}^{-}}{\lambda-s_{j_{n}}^{+}}\right) .
$$

Легко видеть, что $\hat{\alpha}_{-}(\lambda)$ аналитична и не имеет нулей справа от $\Gamma_{\hat{J} ; \hat{K}}^{(n-1)}$ и $\Gamma_{J ; K}^{(n)}$, в то время как $\hat{\alpha}_{+}^{-1}(\lambda)$ аналитична и не имеет нулей слева от $\Gamma_{\hat{J} ; \hat{K}}^{(n-1)}$ и $\Gamma_{J ; K}^{(n)}$. Более того, обе функции $\hat{\alpha}_{ \pm}(\lambda)$ аналитичны и не обращаются в нуль во внутренней области между $\Gamma_{\hat{J} ; \hat{K}}^{(n-1)}$ и $\Gamma_{J ; K}^{(n)}$.

Подставляя (5.11), (5.12) в (5.8) и (5.9), получаем

$$
\begin{aligned}
\iint_{\Gamma_{\hat{J} ; \hat{K}}^{(n-1)}} \frac{\nu(\lambda) \nu(\mu)}{\left(\lambda-\mu_{+}\right)^{2}} d \lambda d \mu= & -\frac{1}{2 \pi i} \int_{\Gamma_{\hat{J} ; \hat{K}}^{(n-1)}} \ln ^{\prime} \hat{\alpha}_{+}(\mu) \cdot \ln \hat{\alpha}_{-}(\mu) d \mu+ \\
& +\ln \left(\frac{\alpha_{-}\left(r_{k_{n}}^{-} ; n-1\right) \hat{\alpha}_{+}\left(s_{j_{n}}^{+}\right)}{\alpha_{-}\left(s_{k_{n}}^{-} ; n-1\right) \hat{\alpha}_{+}\left(r_{j_{n}}^{+}\right)}\right) \\
\iint_{\Gamma_{J ; K}^{(n)}} \frac{\nu(\lambda) \nu(\mu)}{\left(\lambda-\mu_{+}\right)^{2}} d \lambda d \mu= & -\frac{1}{2 \pi i} \int_{\Gamma_{J ; K}^{(n)}} \ln ^{\prime} \hat{\alpha}_{+}(\mu) \cdot \ln \hat{\alpha}_{-}(\mu) d \mu+ \\
& +\ln \left(\frac{\alpha_{-}\left(r_{k_{n}}^{-} ; n\right) \hat{\alpha}_{+}\left(s_{k_{n}}^{-}\right)}{\alpha_{-}\left(s_{j_{n}}^{+} ; n\right) \hat{\alpha}_{+}\left(r_{j_{n}}^{+}\right)}\right) .
\end{aligned}
$$

Пользуясь тем, что функция $\ln \hat{\alpha}_{ \pm}(\mu)$ голоморфна во внутренней области между $\Gamma_{\hat{J} ; \hat{K}}^{(n-1)}$ и $\Gamma_{J ; K}^{(n)}$, находим

$$
\left(\iint_{\Gamma_{J ; K}^{(n)}}-\iint_{\Gamma_{\hat{J} ; \hat{K}}^{(n-1)}}\right) \frac{\nu(\lambda) \nu(\mu)}{\left(\lambda-\mu_{+}\right)^{2}} d \lambda d \mu=\ln \left(\frac{\alpha_{-}\left(s_{k_{n}}^{-} ; n-1\right) \cdot \alpha_{-}\left(r_{k_{n}}^{-} ; n\right) \cdot \hat{\alpha}_{+}\left(s_{k_{n}}^{-}\right)}{\alpha_{-}\left(r_{k_{n}}^{-} ; n-1\right) \cdot \alpha_{-}\left(s_{j_{n}}^{+} ; n\right) \cdot \hat{\alpha}_{+}\left(s_{j_{n}}^{+}\right)}\right) .
$$


Последний шаг состоит в том, чтобы выразить полученный ответ в терминах функций $\alpha_{ \pm}(\lambda ; n-1)$ с помощью (5.10)-(5.12). Нам также потребуются следующие очевидные соотношения:

$$
\begin{aligned}
\hat{\alpha}_{-}\left(s_{j_{n}}^{+}\right) & =\left.\left(s_{j_{n}}^{+}-r_{j_{n}}^{+}\right) \frac{d}{d \lambda} \alpha_{-}(\lambda ; n-1)\right|_{\lambda=s_{j_{n}}^{+}}, \\
\hat{\alpha}_{+}^{-1}\left(s_{k_{n}}^{-}\right) & =\left.\left(s_{k_{n}}^{-}-r_{k_{n}}^{-}\right) \frac{d}{d \lambda} \alpha_{+}^{-1}(\lambda ; n-1)\right|_{\lambda=s_{k_{n}}^{-}}
\end{aligned}
$$

И

$$
\begin{aligned}
\gamma F^{\prime}\left(s_{j_{n}}^{+}\right) & =\left.\alpha_{+}^{-1}\left(s_{j_{n}}^{+} ; n-1\right) \frac{d}{d \lambda} \alpha_{-}(\lambda ; n-1)\right|_{\lambda=s_{j_{n}}^{+}}, \\
\gamma F^{\prime}\left(s_{k_{n}}^{-}\right) & =\left.\alpha_{-}\left(s_{k_{n}}^{-} ; n-1\right) \frac{d}{d \lambda} \alpha_{+}^{-1}(\lambda ; n-1)\right|_{\lambda=s_{k_{n}}^{-}} .
\end{aligned}
$$

Подставляя эти формулы в (5.15), мы получаем после несложных преобразований

$$
\left(\iint_{\Gamma_{J ; K}^{(n)}}-\iint_{\Gamma_{\hat{J} ; \hat{K}}^{(n-1)}}\right) \frac{\nu(\lambda) \nu(\mu)}{\left(\lambda-\mu_{+}\right)^{2}} d \lambda d \mu=\ln \left(\frac{\alpha_{-}^{2}\left(s_{k_{n}}^{-} ; n-1\right) \alpha_{+}^{-2}\left(s_{j_{n}}^{+} ; n-1\right)}{\left(s_{j_{n}}^{+}-s_{k_{n}}^{-}\right)^{2} \gamma^{2} F^{\prime}\left(s_{j_{n}}^{+}\right) F^{\prime}\left(s_{k_{n}}^{-}\right)}\right) .
$$

Объединяя этот результат с (5.4), мы приходим к утверждению леммы.

СлеДСтвиЕ. Пусть $\alpha(\lambda)$ определена формулой (2.1). Тогда

$$
\begin{aligned}
& \exp \left\{\mathcal{A}_{\Gamma_{J ; K}^{(n)}}([g],[\nu])-\mathcal{A}_{\mathbb{R}}([g],[\nu])\right\}= \\
& =\left(\operatorname{det} \frac{1}{s_{j_{a}}^{+}-s_{k_{b}}^{-}}\right)^{2} \prod_{m=1}^{n}\left(\frac{\alpha_{-}\left(s_{k_{m}}^{-}\right)}{\alpha_{+}\left(s_{j_{m}}^{+}\right)}\right)^{2} \frac{e_{+}^{2}\left(s_{j_{m}}^{+}\right) e_{-}^{2}\left(s_{k_{m}}^{-}\right)}{\gamma^{2} F^{\prime}\left(s_{j_{m}}^{+}\right) F^{\prime}\left(s_{k_{m}}^{-}\right)} .
\end{aligned}
$$

ДокАзАТЕльСтво. Применяя последовательно лемму к экспонентам

$$
\exp \left\{\mathcal{A}_{\Gamma_{j_{1}, \ldots, j_{m} ; k_{1}, \ldots, k_{m}}^{(m)}}([g],[\nu])-\mathcal{A}_{\Gamma_{j_{1}, \ldots, j_{m-1} ; k_{1}, \ldots, k_{m-1}}^{(m-1)}}([g],[\nu])\right\}
$$

для $m=1, \ldots, n$, имеем

$$
\begin{aligned}
\exp \left\{\mathcal{A}_{\Gamma_{J ; K}^{(n)}}-\mathcal{A}_{\mathbb{R}}\right\}= & \prod_{m=1}^{n}\left(\frac{\alpha_{-}\left(s_{k_{m}}^{-} ; \Gamma_{j_{1}, \ldots, j_{m-1} ; k_{1}, \ldots, k_{m-1}}^{(m-1)}\right)}{\alpha_{+}\left(s_{j_{m}}^{+} ; \Gamma_{j_{1}, \ldots, j_{m-1} ; k_{1}, \ldots, k_{m-1}}^{(m-1)}\right)}\right)^{2} \times \\
& \times \frac{e_{+}^{2}\left(s_{j_{m}}^{+}\right) e_{-}^{2}\left(s_{k_{m}}^{-}\right)}{\left(s_{j_{m}}^{+}-s_{k_{m}}^{-}\right)^{2} \gamma^{2} F^{\prime}\left(s_{j_{m}}^{+}\right) F^{\prime}\left(s_{k_{m}}^{-}\right)} .
\end{aligned}
$$

Из формулы (5.10) следует, что

$$
\alpha_{ \pm}\left(\lambda ; \Gamma_{j_{1}, \ldots, j_{m-1} ; k_{1}, \ldots, k_{m-1}}^{(m-1)}\right)=\alpha_{ \pm}(\lambda) \prod_{a=1}^{m-1}\left(\frac{\lambda-s_{k_{a}}^{-}}{\lambda-s_{j_{a}}^{+}}\right) .
$$

Остается подставить это в (5.20) и воспользоваться равенством

$$
\operatorname{det}_{\substack{j_{1}, \ldots, j_{n} \\ k_{1}, \ldots, k_{n}}} \frac{1}{s_{j_{a}}^{+}-s_{k_{b}}^{-}}=\frac{\prod_{a>b}^{n}\left(s_{j_{a}}^{+}-s_{j_{b}}^{+}\right)\left(s_{k_{b}}^{-}-s_{k_{a}}^{-}\right)}{\prod_{a, b=1}^{n}\left(s_{j_{a}}^{+}-s_{k_{b}}^{-}\right)} .
$$

Следствие доказано. 
ДокАЗАТЕЛЬСТво тЕОРЕмы 2.3. Асимптотическое поведение детерминанта Фредгольма (4.10) содержит определитель $\operatorname{det}(I-A)$ с $A=A^{-} A^{+}$. Пусть для определенности $n_{+} \leqslant n_{-}$(в противном случае в силу (4.12) $\operatorname{det}(I-A)$ может быть заменен на $\operatorname{det}(I-\tilde{A})$ с $\left.\tilde{A}=A^{+} A^{-}\right)$. Тогда имеем

$$
\operatorname{det}(I-A)=\sum_{n=0}^{n_{+}} \sum_{j_{n}>\cdots>j_{1}}^{n_{+}} \operatorname{det}_{a, b=1, \ldots, n}\left(-\sum_{k=1}^{n_{-}} A_{j_{a}, k}^{-} A_{k, j_{b}}^{+}\right) .
$$

Подставляя сюда $A^{ \pm}$из (2.4), получаем

$$
\operatorname{det}(I-A)=\sum_{n=0}^{n_{+}} \sum_{n_{n}}^{n_{+}} \sum_{j_{+}}^{n_{-}} \sum_{j_{1}}^{n} \frac{h_{k_{a}}^{-} h_{j_{a}}^{+} e_{-}^{2}\left(s_{k_{a}}^{-}\right) e_{+}^{2}\left(s_{j_{a}}^{+}\right)}{s_{j_{a}}^{+}-s_{k_{a}}^{-}} \underset{a, b=1, \ldots, n}{\operatorname{det}}\left(\frac{1}{s_{j_{a}}^{+}-s_{k_{b}}^{-}}\right) .
$$

Остается использовать явные выражения (2.4) для $h^{ \pm}$и совершить замену

$$
\prod_{a=1}^{n}\left(s_{j_{a}}^{+}-s_{k_{a}}^{-}\right)^{-1} \mapsto \frac{1}{n !} \operatorname{det}_{a, b=1, \ldots, n}\left(\frac{1}{s_{j_{a}}^{+}-s_{k_{b}}^{-}}\right) .
$$

Такая замена возможна, поскольку по всем $j_{\ell}$ и $k_{\ell}$ ведется суммирование. Тогда в силу (5.19) мы приходим к равенству

$$
\operatorname{det}(I-A)=\sum_{n=0}^{n_{+}} \sum_{j_{n}>\cdots>j_{1}}^{n_{+}} \sum_{k_{n}>\cdots>k_{1}}^{n_{-}} \exp \left\{\mathcal{A}_{\Gamma_{J ; K}^{(n)}}([g],[\nu])-\mathcal{A}_{\mathbb{R}}([g],[\nu])\right\} .
$$

Принимая во внимание (2.10), мы получаем утверждение теоремы.

\section{6. ПРИМЕРЫ}

В этом разделе мы рассмотрим приложения полученных результатов.

\section{1. Температурная корреляционная функция непроницаемых бозонов.} Оператор числа частиц $Q_{x}$ на отрезке $[0, x]$ играет важную роль в теории квантовых одномерных интегрируемых систем [29]-[31]. Среднее от $e^{\beta Q_{x}}$, где $\beta$ - комплексный параметр, является производящей функцией для некоторых корреляционных функций таких моделей. В модели непроницаемых бозонов при конечной температуре это среднее равно детерминанту Фредгольма оператора $I+\gamma V(1.2)$ с $g(\lambda)=0$ и

$$
\gamma F(\lambda)=\frac{e^{\beta}-1}{e^{\left(\lambda^{2}-h\right) / T}+1},
$$

где $h$ - химический потенциал и $T$ - температура. Главные члены асимптотического разложения этого детерминанта при большом $x$ были вычислены в работах [19], [20], [31]. Формулы (2.10), (2.11) дают полное асимптотическое разложение. При этом

$$
\nu(\lambda)=-\frac{1}{2 \pi i} \ln \left(\frac{e^{\left(\lambda^{2}-h\right) / T}+e^{\beta}}{e^{\left(\lambda^{2}-h\right) / T}+1}\right),
$$


и функция $\alpha(\lambda)$ задана формулой (2.1). Корни уравнения $1+\gamma F(\lambda)=0$ образуют две серии $s_{j, i}^{ \pm}, i=1,2$ :

$$
\begin{array}{ll}
s_{j, 1}^{+}=\sqrt{h+\beta T+i \pi T(2 j+1)}, & s_{j, 2}^{+}=-\left(s_{j, 1}^{+}\right)^{*}, \\
s_{j, 1}^{-}=\left(s_{j, 1}^{+}\right)^{*}, & s_{j, 2}^{-}=-\left(s_{j, 1}^{-}\right)^{*}, \quad j=0,1, \ldots .
\end{array}
$$

Формулы (2.10), (2.11) справедливы для произвольного $N=n_{+}=n_{-}$. При фиксированном $N$ мы пренебрегаем корнями $s_{N+1}^{ \pm}$, дающими вклады порядка $e^{i x\left(s_{N+1, j}^{+}-s_{1, k}^{-}\right)}$. Поэтому в остаточном члене $O\left(e^{-a x}\right)$ можно положить $a=\operatorname{Im}\left(s_{N+1,1}^{+}-s_{1,1}^{-}\right)$.

6.2. Нормировочный множитель в спиновой $X X Z$-цепочке. Наш второй пример связан с $X X Z$-цепочкой Гейзенберга спина $1 / 2$ во внешнем магнитном поле. В термодинамическом пределе корреляционные функции этой модели содержат нормировочный множитель, пропорциональный детерминанту Фредгольма $\operatorname{det}_{[-x / 2, x / 2]}(I+K)[32]$, где оператор $K\left(t-t^{\prime}\right)$ действует на интервале [-x/2,x/2] и имеет ядро

$$
K\left(t-t^{\prime}\right)=\frac{\sin 2 \zeta}{2 \pi \operatorname{sh}\left(t-t^{\prime}-i \zeta\right) \operatorname{sh}\left(t-t^{\prime}+i \zeta\right)} .
$$

Здесь $0<\zeta<\pi$ и $\cos \zeta=\Delta$, где $\Delta$ - параметр анизотропии модели. Длина интервала $x$ зависит от величины внешнего магнитного поля. Если последнее стремится к нулю, то $x \rightarrow \infty$.

Оператор $I+K$ принадлежит классу обрезанных операторов Винера-Хопфа. Они могут быть сведены к операторам с ОСЯ с помощью преобразования Фурье. Пусть $\chi_{[-x / 2, x / 2]}\left(t^{\prime}\right)$ является характеристической функцией интервала $[-x / 2, x / 2]$. Тогда

$$
\operatorname{det}_{[-x / 2, x / 2]}(I+K)=\operatorname{det}_{\mathbb{R}}(I+V)
$$

где оператор $I+V$ действует на $\mathbb{R}$ и

$$
V(\lambda, \mu)=\frac{1}{2 \pi} \int_{\mathbb{R}} e^{i t \lambda} K\left(t-t^{\prime}\right) \chi_{[-x / 2, x / 2]}\left(t^{\prime}\right) e^{-i t^{\prime} \mu} d t d t^{\prime} .
$$

Вычисляя этот интеграл, находим

$$
V(\lambda, \mu)=F(\lambda) \frac{\sin \frac{x}{2}(\lambda-\mu)}{\pi(\lambda-\mu)},
$$

где

$$
F(\lambda)=\widehat{K}(\lambda)=\frac{\operatorname{sh}[\lambda(\pi / 2-\zeta)]}{\operatorname{sh}[\lambda \pi / 2]} .
$$

Таким образом, с точностью до преобразования подобия мы получаем ОСЯ с $g(\lambda)=0$ и $F(\lambda)$, заданной в (6.8). Следовательно, при достаточно большом $x$ (что соответствует малому магнитному полю) мы можем асимптотически вычислить исходный детерминант $\operatorname{det}_{[-x / 2, x / 2]}(I+K)$. 
Как и в предыдущем примере, формулы (2.10), (2.11) дают полное асимптотическое разложение. Имеем

$$
\nu(\lambda)=-\frac{1}{2 \pi i} \ln \left(\frac{2 \operatorname{sh}[\lambda(\pi-\zeta) / 2] \operatorname{ch}[\lambda \zeta / 2]}{\operatorname{sh}[\lambda \pi / 2]}\right)
$$

Функция $\alpha(\lambda)$ может быть явно найдена в терминах $Г$-функций:

$$
\alpha_{-}(\lambda)=\sqrt{2(\pi-\zeta)}\left(\frac{\pi}{\zeta}\right)^{-\frac{i \lambda \zeta}{2 \pi}}\left(\frac{\pi}{\pi-\zeta}\right)^{-\frac{i \lambda(\pi-\zeta)}{2 \pi}} \frac{\Gamma\left(1+\frac{i \lambda}{2}\right)}{\Gamma\left(\frac{1}{2}+\frac{i \lambda \zeta}{2 \pi}\right) \Gamma\left(1+\frac{i \lambda(\pi-\zeta)}{2 \pi}\right)}
$$

и $\alpha_{+}^{-1}(\lambda)=\alpha_{-}(-\lambda)$. Аналогично случаю, рассмотренному выше, корни уравнения $1+\gamma F(\lambda)=0$ образуют две серии $s_{j, i}^{ \pm}, i=1,2$. Если $\pi / \zeta$ не является рациональным числом, то

$$
s_{j, 1}^{+}=\frac{2 \pi i}{\pi-\zeta}(j+1), \quad s_{j, 2}^{+}=\frac{\pi i}{\zeta}(2 j+1), \quad s_{j, i}^{-}=\left(s_{j, i}^{+}\right)^{*}, \quad j=0,1, \ldots .
$$

Если $\pi / \zeta$ - рациональное число, то в (6.11) следует пропустить те $s_{j, i}^{ \pm}$, которые удовлетворяют условию $\operatorname{sh}\left(\pi s_{j, i}^{ \pm} / 2\right)=0$. Асимптотические оценки $(2.10),(2.11)$ справедливы для произвольного $N=n_{+}=n_{-}$. Аналогично рассмотренному выше случаю в остаточном члене $O\left(e^{-a x}\right) \quad$ пожно положить $a=$ $\min _{i, i^{\prime}=1,2} \operatorname{Im}\left(s_{N+1, i}^{+}-s_{1, i^{\prime}}^{-}\right)$.

Благодарности. Я благодарен Н. Китанину, К. К. Козловскому, Ж.-М. Майе и В. Террас за полезные дискуссии. Эта работа была частично поддержана Франко-Российской организацией по теоретической и математической физике (гранты GDRI-471 CNRS и РФФИ-09-01-93106-НЦНИЛ_а), Программой РАН "Математические методы нелинейной динамики", РФФИ (гранты № 08-01-00501_a, 09-01-12150-офи_м), Программой поддержки ведущих научных школ (грант НШ-8265.2010.1).

\section{Список литературы}

[1] M. Gaudin, Nucl. Phys., 25 (1961), 447-458.

[2] M. L. Mehta, M. Gaudin, Nucl. Phys., 18 (1960), 420-427.

[3] A. Lenard, J. Math. Phys., 5:7 (1964), 930-943.

[4] M. Jimbo, T. Miwa, Y. Môri, M. Sato, Physica D, 1:1 (1980), 80-158.

[5] J. des Cloizeaux, M. L. Mehta, J. Math. Phys., 14:11 (1973), 1648-1650.

[6] H. Widom, Indiana Univ. Math. J., 21 (1971), 277-283.

[7] F. Dyson, Comm. Math. Phys., 47:2 (1976), 171-183.

[8] H. Widom, J. Approx. Theory, 77:1 (1994), 51-64.

[9] H. Widom, Comm. Math. Phys., 171:1 (1995), 159-180, arXiv: hep-th/9405010.

[10] V. I. Krasovsky, Int. Math. Res. Not., 25 (2004), 1249-1272.

[11] T. Ehrhardt, Comm. Math. Phys., 262:2 (2006), 317-341.

[12] P. A. Deift, A. R. Its, I. Krasovsky, X. Zhou, J. Comput. Appl. Math., 202:1 (2007), 26-47, arXiv: math/0601535.

[13] А. М. Будылин, В. С. Буслаев, Алгебра и анализ, 7:6 (1995), 79-103.

[14] P. A. Deift, A. R. Its, X. Zhou, Ann. Math., 146:1 (1997), 149-235.

[15] В. А. Фок, Матем. сб., 14:1-2 (1944), 3-50. 
[16] Б. Нобл, Применение метода Винера-Хопфа для решения дифференииалъных уравнений в частных производных, ИЛ, М., 1962.

[17] B. M. McCoy, J. H. H. Perk, R. E. Shrock, Nucl. Phys. B, 220:1 (1983), 35-47.

[18] A. Lenard, J. Math. Phys., 7:7 (1966), 1268-1272.

[19] A. R. Its, A. G. Izergin, V. E. Korepin, Comm. Math. Phys., 129:1 (1990), 205-222.

[20] A. R. Its, A. G. Izergin, V. E. Korepin, Comm. Math. Phys., 130:3 (1990), 471-488.

[21] F. Colomo, A. G. Izergin, V. E. Korepin, V. Tognetti, Phys. Lett. A, 169:4 (1992), 243-247.

[22] F. Colomo, A. G. Izergin, V. E. Korepin, V. Tognetti, TMФ, 94:1 (1993), 19-51.

[23] A. R. Its, A. G. Izergin, V.E. Korepin, N. A. Slavnov, Phys. Rev. Lett., 70:11 (1993), 1704-1706; Erratum: 70:15, 2357-2357, arXiv: hep-th/9212135.

[24] A. R. Its, A. G. Izergin, V.E. Korepin, N. A. Slavonv, Internat J. Modern Phys. B, 4:5 (1990), 1003-1037.

[25] V.V. Cheianov, M.R. Zvonarev, J. Phys. A, 37:6 (2004), 2261-2297, arXiv: cond-mat/0310499.

[26] N. Kitanine, K. K. Kozlowski, J.-M. Maillet, N. A. Slavnov, V. Terras, Comm. Math. Phys., 291:3 (2009), 691-761, arXiv: 0805.4586.

[27] P. Deift, "Integrable operators", Differential Operators and Spectral Theory, M.Sh. Birman's 70th anniversary collection, Amer. Math. Soc. Transl. Ser. 2, 189, eds. V. Buslaev, M. Solomyak, D. Yafaev, AMS, Providence, R.I., 1999, 69-84.

[28] Н. И. Ахиезер, Укр. матем. журн., 16 (1964), 445-462.

[29] A. G. Izergin, V. E. Korepin, Comm. Math. Phys., 94:1 (1984), 67-92.

[30] A. G. Izergin, V. E. Korepin, Comm. Math. Phys., 99:2 (1985), 271-302.

[31] V.E. Korepin, N. M. Bogoliubov, A. G. Izergin, Quantum Inverse Scattering Method and Correlation Functions, Cambridge Monogr. Math. Phys., Cambridge Univ. Press, Cambridge, 1993.

[32] V.E. Korepin, Comm. Math. Phys., 86:3 (1982), 391-418. 